\section{Larynxmaske reduziert Inzidenz respiratorischer Komplikationen bei Kindern}

Drake-Brockman TF et al. The effect of endotracheal tubes versus laryngeal mask airways on perioperative respiratory adverse events in infants: a randomised controlled trial. Lancet 2017; 389: $701-708$

Bei Kindern < 1 Jahr mit kleinem operativen Eingriff treten bei Verwendung einer Larynxmaske zur Sicherung des Atemwegs signifikant weniger perioperative respiratorische Komplikationen auf als bei Verwendung eines endotrachealen Tubus, und auch das Auftreten einer schwerwiegenden Komplikation (Laryngospasmus oder Bronchospasmus) ist signifikant seltener. Dies sind die $\mathrm{Er}$ gebnisse einer Untersuchung von Thomas F. E. Drake-Brockman und Kollegen aus dem Princess Margaret Hospital for Children in Perth, Australien.

In einer randomisierten, kontrollierten Single-Center-Studie überprüften sie ihre Hypothese, dass bei Kindern $<1$ Jahr mit kleinem operativen Eingriff 15\% weniger perioperative respiratorische Komplikationen auftreten, wenn eine Larynxmaske anstelle eines endotrachealen Tubus verwendet wird. Unter perioperative respiratorische Komplikationen zählten sie Laryngospasmus und Bronchospasmus als schwerwiegende Komplikationen und außerdem eine Sauerstoffsättigung von <95\%, Atemwegsobstruktionen, schwere Hustenanfälle und postoperativen Stridor. Primärer Endpunkt der Studie war das Auftreten einer der genannten respiratorischen Komplikation intra- oder postoperativ (von Narkoseinduktion bis zur Entlassung aus dem Aufwachraum), sekundäre Endpunkte waren das Auftreten und die Häufigkeit der einzelnen respiratorischen Komplikationen sowie das zeitliche In-Erscheinung-Treten. Sie berechneten eine Studiengröße von 145 Kindern pro Gruppe (Larynxmaske oder Tubus), um eine 80 \%ige Power zu erhalten. Nach Einschluss der Hälfte der hierfür er- forderlichen Patienten planten die Wissenschaftler eine Zwischenanalyse und ein vorzeitiges Beenden der Studie, falls sich bis hierhin ein mehr als 25\%iger Unterschied der primären Outcome-Variablen zwischen den Gruppen zeigen sollte.

Kriterium für die Aufnahme in die Studie war u.a., dass eine kombinierte allgemeine und lokale oder regionale Anästhesie geplant war, sodass Fentanyl nicht in einer Dosierung von $>1 \mu \mathrm{g}$ pro kg Körpergewicht gegeben werden musste. Ansonsten waren weder die Durchführung der Narkose noch die Insertion der Atemwegshilfe standardisiert. Nur für die Ventilation gab es folgende Standardisierung: Pressure-Support-Modus mit $10 \mathrm{cmH} 2 \mathrm{O}$ und einem positiven endexpiratorischen Druck (PEEP) von $5 \mathrm{cmH} 2 \mathrm{O}$.

So schlossen die Untersucher auf randomisiertem Weg zunächst 83 Patienten in die Gruppe „Larynxmaske“ und $94 \mathrm{~Pa}$ tienten in die Gruppe „endotrachealer Tubus“ ein. Die häufigsten Operationen waren dabei Herniotomien, Zystoskopien, MRT- und CT-Untersuchungen sowie kleinere urologische Eingriffe.

Bei der Zwischenanalyse verzeichneten die Untersucher 65 Fälle von perioperativen respiratorischen Komplikationen wie oben beschrieben (=37\% aller untersuchten Kinder), davon traten 15 in der Gruppe „Larynxmaske“ (=18\%) und 50 in der Gruppe „endotrachealer Tubus“ (=53\%) auf. Die Risk-Ratio beträgt demnach 2,94 (Cl 95\%: 1,79-4,83, $\mathrm{p}<$ $0,0001)$. Diese fast 3-fach erhöhte Wahrscheinlichkeit, perioperativ eine respiratorische Komplikation zu erleiden, in der Tubus-Gruppe im Vergleich zur Larynxmaske-Gruppe führte zur vorzeitigen Beendigung der Untersuchung. Und zwar zeigte sich hierbei jede einzelne der respiratorischen Komplikationen in einer höheren Rate in der Tubus- als in der Larynxmaske-Gruppe, und das sowohl intra- als auch postoperativ. Auch schwerwiegende Komplikationen (Laryngooder Bronchospasmus) waren in der Tubusgruppe mit 18 Fällen (=19\%) signifikant häufiger als in der LarynxmaskeGruppe mit 3 Fällen (=4\%), p=0,002.
In der Tubus-Gruppe erhielten (nur) 18 Kinder eine neuromuskuläre Blockade zur Intubation (=19\%), in der Larynxmaske-Gruppe war dies nicht erforderlich. Hinsichtlich des Zeitpunkts der Extubation unterschieden sich die beiden Gruppen: Bei $73 \%$ der Kinder mit Larynxmaske erfolgte die Entfernung in tiefer Anästhesie und bei $20 \%$ wach (bei $6 \%$ existierten zum Zeitpunkt der Extubation keine Daten), aber nur 32\% der Kinder mit endotrachealem Tubus wurden in tiefer Narkose extubiert und $60 \%$ wach.

In einem Fall musste eine Larynxmaske aufgrund von Undichtigkeit gegen einen endotrachealen Tubus ausgetauscht werden, dies geschah noch während der Narkoseeinleitung. In allen anderen Fällen war eine gute Ventilation über Larynxmaske möglich.

FAZIT

Zusammenfassend deutet die vorliegende Untersuchung auf einen Vorteil für die Verwendung einer Larynxmaske im Vergleich zu einem endotrachealen Tubus bei sehr kleinen Kindern im Hinblick auf respiratorische Komplikationen hin. Dieses Ergebnis ist nicht sehr verwunderlich. Aber nicht immer ist der Verzicht auf eine endotracheale Intubation zur Ventilation möglich. Wichtig wären großangelegte Studien, die auch die Risiken von Fehlpositionierung und Aspiration bei diesen sehr kleinen Patienten untersuchen. Wenn kein erhöhtes Aspirationsrisiko besteht, kann laut Autoren das Risiko von respiratorischen Komplikationen durch die Verwendung einer Larynxmaske signifikant minimiert werden.

Dr. med. Sina Pulz, Köln 